\title{
NOTES ON THE COMPUTATION OF LAPLACE-STIELJES TRANSFORM FOR LOGNORMAL AND WEIBULL
}

\author{
Jau-Chuan $\mathrm{Ke}^{1}$, Chia-Huang $\mathrm{Wu}^{2}$ and Wen Lea Pearn ${ }^{2}$ \\ ${ }^{1}$ Department of Applied Statistics \\ National Taichung Institute of Technology \\ Taichung, Taiwan, ROC \\ jauchuan@ntit.edu.tw \\ ${ }^{2}$ Department of Industrial Engineering and Management \\ National Chiao Tung University, Taiwan, ROC \\ \{duckboy614583@gmail.com\} \\ \{wlpearn@mail.nctu.edu.tw\}
}

\begin{abstract}
In this paper, we perform the computation of the Laplace-Stieljes transform of Lognormal and Weibull distribution, in which the upper limit of the definite integral from infinite to 1 by using proper transformation. Some advantages are found in this study.
\end{abstract}

Key Words- Laplace-Stieljes transform; Lognormal; Weibull

\section{INTRODUCTION}

In reliability/availability models, both Lognormal and Weibull distribution play important roles. The system measures are usually expressed as Laplace-Stieljes transform (LST) of failure/repair time in such models. Unfortunately, the explicitly closed forms of the LST of the Lognormal and Weibull are not existed (see Trivedi [1] and Casella and Berger [2]). Therefore, they are not easily to be implemented without mathematical software (such as Maple, Matlab, etc). The readers can refer to the recently published papers, such as Wang and Chiu [3], Wang and Chen [4] and Ke et al. [5]. To conquer this problem, the computable forms of the LST of Lognormal and Weibull are derived in this paper.

Wang and Chiu [3] investigated the cost benefit of availability system with warm standby units and imperfect coverage, where three models are considered. In Wang and Chiu's study [3], they calculated the explicit form of availability of three models, and achieved the comparison of all models based on availability and cost/benefit ratios 
criterion with three different repair time distributions which are exponential, 3-Erlang, and deterministic. Nevertheless, the Lognormal and Weibull distributions are not considered in this paper due to the difficulty in integral computation of their LST.

The aim of this paper is that an efficient reciprocal transformation is tried to improve the computation time spent for the integral computation of their LST. Firstly, we derive a new Laplace-Stieljes transform as definite integral from zero to one. Secondly, we discuss the availability of these three models by Wang and Chiu [3] when the repair time is Lognormal or Weibull distribution. Finally, we make some comparisons on the computation time spent between the integral formula from zero to infinity and reciprocal transformation developed in this paper (by MAPLE software).

\section{TRANSFORMATION COMPUTATION OF LST}

The LST of $\mathrm{X}$ is as

$$
B^{*}(s)=\int_{0}^{\infty} e^{-s x} f_{X}(x) d x .
$$

The computation listed above, the upper limit of the definite integral is infinite which it is cumbersome if one doesn't adopt by mathematical software. Making the reciprocal transformation of $y=1 /(1+x)$, the LST can be rewritten as

$$
B^{*}(s)=\int_{0}^{1} \exp \left(-s\left(\frac{1}{y}-1\right)\right) f_{X}\left(\frac{1}{y}-1\right) \frac{1}{y^{2}} d y .
$$

(i) If the random variable $X \sim$ exponential with mean $1 / \mu$, the probability density

function is

$$
f_{X}(x)=\mu e^{-\mu x}
$$

We have

$$
B^{*}(s)=\int_{0}^{1} \frac{\mu}{y^{2}} e^{-(s+\mu)\left(\frac{1}{y}-1\right)} d y=\frac{\mu}{\mu+s},
$$

which is accordance with that of Trivedi [1].

(ii) If the random variable $X \sim k$-stage Erlang, each with mean $1 / k \mu$, the probability density function is

$$
f_{X}(x)=\frac{(k \mu)^{k}}{\Gamma(k)} x^{k-1} e^{-k \mu x}
$$

We have

$$
B^{*}(s)=\int_{0}^{1} \frac{(k \mu)^{k}(1-y)^{k-1}}{\Gamma(k) y^{k+1}} e^{-(s+k \mu)\left(\frac{1}{y}-1\right)} d y=\left(\frac{k \mu}{k \mu+s}\right)^{k},
$$

which is consistent with that of Trivedi [1]. 
(iii) If the random variable $X$ is deterministic, equal to $1 / \mu$

$$
f_{X}(x)=\frac{1}{\mu} \text { with probability } 1 .
$$

Then we have

$$
B^{*}(s)=e^{-s / \mu},
$$

which is consistent with that of Trivedi [1].

(iv) If the random variable $X \sim \operatorname{LogN}\left(\mu, \sigma^{2}\right)$, the probability density function is

$$
f_{X}(x)=\frac{1}{\sqrt{2 \pi} \sigma x} e^{-\frac{(\ln (x)-\mu)^{2}}{2 \sigma^{2}}},
$$

which leads to

$$
B^{*}(s)=\int_{0}^{1} e^{-s\left(\frac{1}{y}-1\right)} \frac{1}{\sqrt{2 \pi} \sigma y(1-y)} e^{-\frac{(\ln (1-y)-\ln (y)-\mu)^{2}}{2 \sigma^{2}}} d y .
$$

(v) If the random variable $X \sim \operatorname{Weibull}(a, b)$, the probability density function is

$$
f_{X}(x)=a b x^{b-1} e^{-a x^{b}},
$$

which implies

$$
B^{*}(s)=\int_{0}^{1} a b \frac{(1-y)^{b-1}}{y^{b+1}} e^{-\left(s\left(\frac{1-y}{y}\right)+a\left(\frac{1-y}{y}\right)^{b}\right)} d y .
$$

It should be noted that when no mathematical software is used, the computation of $\int_{0}^{1} f(\cdot) d x$ is more easy and stable than $\int_{0}^{\infty} f(\cdot) d x$. Also, the computation time of $\int_{0}^{1} f(\cdot) d x$ is much less than $\int_{0}^{\infty} f(\cdot) d x$.

\section{EXAMPLE ILLUSTRATION}

To demonstrate the validness of this reciprocal transformation, the four cases in Wang and Chiu [3] are considered to perform a comparative analysis of the $A v$ for the availability models 1,2 , and 3 when the repair time distribution is exponential $(M), 3-$ stage Erlang $\left(E_{3}\right)$, deterministic $(D)$, Lognormal ( $\left.L N\right)$, and Weibull $(W)$, respectively. In order to make the comparisons, the parameters of Lognormal (Weibull) distribution are setting $a=-\ln (\mu)-1 / 2$ and $b=1\left(a=\pi \mu^{2} / 4\right.$ and $\left.b=2\right)$ such that the expected repair time equals $1 / \mu$ (as Wang and Chiu [3]). All numerical results are obtained by the mathematical program MAPLE 9, which are based on the equipment of the following setting: CPU-Pentium 4 3.0 GHz, RAM 1.50 GB.

Numerical results of the $A v_{i}(M), A v_{i}\left(E_{3}\right), A v_{i}(D), A v_{i}(L N)$, and $A v_{i}(W)$ for each availability model $i(i=1,2,3)$ are shown in Tables $1-4$ for Cases $1-4$, respectively. The comparative analysis of the cost/benefit ratios is made based on assumed the cost 
models of the models in Wang and Chiu's model [3, pp.1251-1252]. The rank (cost/benefit) are shown in Table 5-8, for ranges of $\lambda, \mu, \beta$, and $c$, respectively.

Table 1. Comparison of the availability models $1,2,3$ for $A v$ (case 1)

\begin{tabular}{ll}
\hline Range of $\lambda$ & Result \\
\hline 1. Exponential repair time $(M)$ & \\
$0.001<\lambda<0.00282$ & $A v_{3}(M)>A v_{1}(M)>A v_{2}(M)$ \\
$0.00282<\lambda<0.01$ & $A v_{1}(M)>A v_{3}(M)>A v_{2}(M)$
\end{tabular}

2. 3-stage Erlang repair time $\left(E_{3}\right)$
$0.001<\lambda<0.00146$
$A v_{1}\left(E_{3}\right)>A v_{3}\left(E_{3}\right)>A v_{2}\left(E_{3}\right)$
$0.00146<\lambda<0.00431$
$A v_{3}\left(E_{3}\right)>A v_{1}\left(E_{3}\right)>A v_{2}\left(E_{3}\right)$
$0.00431<\lambda<0.01$
$A v_{1}\left(E_{3}\right)>A v_{3}\left(E_{3}\right)>A v_{2}\left(E_{3}\right)$

3. Deterministic repair time $(D)$

$$
\begin{aligned}
& 0.001<\lambda<0.00196 \\
& 0.00196<\lambda<0.00583 \\
& 0.00583<\lambda<0.01
\end{aligned}
$$$$
\begin{aligned}
& A v_{1}(D)>A v_{3}(D)>A v_{2}(D) \\
& A v_{3}(D)>A v_{1}(D)>A v_{2}(D) \\
& A v_{1}(D)>A v_{3}(D)>A v_{2}(D)
\end{aligned}
$$

4. Lognormal repair time $(L N)$

$$
0.001<\lambda<0.01
$$

$$
A v_{1}(L N)>A v_{3}(L N)>A v_{2}(L N)
$$

5. Weibull repair time $(W)$
$0.001<\lambda<0.00152$
$A v_{1}(W)>A v_{3}(W)>A v_{2}(W)$
$0.00152<\lambda<0.00464$
$A v_{3}(W)>A v_{1}(W)>A v_{2}(W)$
$0.00464<\lambda<0.01$
$A v_{1}(W)>A v_{3}(W)>A v_{2}(W)$ 
Table 2. Comparison of the availability models $1,2,3$ for $A v$ (case 2)

\begin{tabular}{ll}
\hline Range of $\mu$ & Result \\
\hline 1. Exponential repair time $(M)$ & $A v_{3}(M)>A v_{1}(M)>A v_{2}(M)$ \\
$0.06<\mu<0.10199$ & $A v_{1}(M)>A v_{3}(M)>A v_{2}(M)$ \\
$0.10199<\mu<0.16$ & \\
2. 3-stage Erlang repair time $\left(E_{3}\right)$ & $A v_{3}\left(E_{3}\right)>A v_{1}\left(E_{3}\right)>A v_{2}\left(E_{3}\right)$ \\
$0.06<\mu<0.08751$ & $A v_{1}\left(E_{3}\right)>A v_{3}\left(E_{3}\right)>A v_{2}\left(E_{3}\right)$ \\
$0.08751<\mu<0.16$ & \\
3. Deterministic repair time $(D)$ & $A v_{3}(D)>A v_{1}(D)>A v_{2}(D)$ \\
$0.06<\mu<0.07792$ & $A v_{1}(D)>A v_{3}(D)>A v_{2}(D)$ \\
$0.07792<\mu<0.16$ & \\
4. Lognormal repair time $(L N)$ & $A v_{3}(L N)>A v_{1}(L N)>A v_{2}(L N)$ \\
$0.06<\mu<0.0902$ & $A v_{1}(L N)>A v_{3}(L N)>A v_{2}(L N)$ \\
$0.0902<\mu<0.16$ & \\
5. Weibull repair time $(W)$ & $A v_{3}(W)>A v_{1}(W)>A v_{2}(W)$ \\
$0.06<\mu<0.08618$ & $A v_{1}(W)>A v_{3}(W)>A v_{2}(W)$ \\
$0.06<\mu<0.16$ &
\end{tabular}

\begin{tabular}{ll}
\hline Table 3. Comparison of the availability models $1,2,3$ for $A v$ (case 3) \\
\hline Range of $\beta$ & Result
\end{tabular}

1. Exponential repair time $(M)$

$1<\beta<2.32435$

$2.32435<\beta<10$

2. 3-stage Erlang repair time ( $\left.E_{3}\right)$

$1<\beta<3.03885$

$3.03885<\beta<10$

3. Deterministic repair time $(D)$

$1<\beta<3.79644$

$3.79644<\beta<10$

4. Lognormal repair time ( $L N)$

$1<\beta<2.65343$

$2.65343<\beta<10$

5. Weibull repair time $(W)$

$1<\beta<3.13083$

$3.13083<\beta<10$
$A v_{1}(M)>A v_{3}(M)>A v_{2}(M)$

$A v_{3}(M)>A v_{1}(M)>A v_{2}(M)$

$A v_{1}\left(E_{3}\right)>A v_{3}\left(E_{3}\right)>A v_{2}\left(E_{3}\right)$

$A v_{3}\left(E_{3}\right)>A v_{1}\left(E_{3}\right)>A v_{2}\left(E_{3}\right)$

$A v_{1}(D)>A v_{3}(D)>A v_{2}(D)$

$A v_{3}(D)>A v_{1}(D)>A v_{2}(D)$

$A v_{1}(L N)>A v_{3}(L N)>A v_{2}(L N)$

$A v_{3}(L N)>A v_{1}(L N)>A v_{2}(L N)$

$A v_{1}(W)>A v_{3}(W)>A v_{2}(W)$

$A v_{3}(W)>A v_{1}(W)>A v_{2}(W)$ 
Table 4. Comparison of the availability models $1,2,3$ for $A v$ (case 4)

\begin{tabular}{ll}
\hline Range of $c$ & Result \\
\hline 1. Exponential repair time $(M)$ & \\
$0.5<c<0.89675$ & $A v_{1}(M)>A v_{3}(M)>A v_{2}(M)$ \\
$0.89675<c<1.0$ & $A v_{3}(M)>A v_{1}(M)>A v_{2}(M)$
\end{tabular}

2. 3-stage Erlang repair time $\left(E_{3}\right)$
$0.5<c<0.52111$
$A v_{1}\left(E_{3}\right)>A v_{2}\left(E_{3}\right)>A v_{3}\left(E_{3}\right)$
$0.52111<c<0.92078$
$A v_{1}\left(E_{3}\right)>A v_{3}\left(E_{3}\right)>A v_{2}\left(E_{3}\right)$
$0.92078<c<1.0$
$A v_{3}\left(E_{3}\right)>A v_{1}\left(E_{3}\right)>A v_{2}\left(E_{3}\right)$

3. Deterministic repair time $(D)$

$$
\begin{aligned}
& 0.5<c<0.63348 \\
& 0.63348<c<0.93631 \\
& 0.93631<c<1.0
\end{aligned}
$$

$$
\begin{aligned}
& A v_{1}(D)>A v_{2}(D)>A v_{3}(D) \\
& A v_{1}(D)>A v_{3}(D)>A v_{2}(D) \\
& A v_{3}(D)>A v_{1}(D)>A v_{2}(D)
\end{aligned}
$$

4. Lognormal repair time ( $L N)$

$$
\begin{aligned}
& 0.5<c<0.91011 \\
& 0.91011<c<1.0
\end{aligned}
$$$$
A v_{1}(L N)>A v_{3}(L N)>A v_{2}(L N)
$$$$
A v_{3}(L N)>A v_{1}(L N)>A v_{2}(L N)
$$

5. Weibull repair time $(W)$

$$
\begin{aligned}
& 0.5<c<0.54073 \\
& 0.54073<c<0.92306 \\
& 0.92306<c<1.0
\end{aligned}
$$

$$
\begin{aligned}
& A v_{1}(W)>A v_{2}(W)>A v_{3}(W) \\
& A v_{1}(W)>A v_{3}(W)>A v_{2}(W) \\
& A v_{3}(W)>A v_{1}(W)>A v_{2}(W)
\end{aligned}
$$

Table 5. Rank of $C_{i} / A v_{i}$ for $\alpha=0.0005, \mu=0.1, \beta=2.4, c=0.9$

\begin{tabular}{lcc}
\hline Repair time distribution & Range of $\lambda$ & Rank \\
\hline Exponential $(M)$ & $0.001<\lambda<0.01$ & Model 2 \\
3-stage Erlang $\left(E_{3}\right)$ & $0.001<\lambda<0.01$ & Model 2 \\
Deterministic $(D)$ & $0.001<\lambda<0.01$ & Model 2 \\
Lognormal $(L N)$ & $0.001<\lambda<0.01$ & Model 2 \\
Weibull $(W)$ & $0.001<\lambda<0.01$ & Model 2 \\
\hline Table 6. Rank of $C_{i} / A v_{i}$ for $\lambda=0.001, \alpha=0.0005, \beta=2.4, c=0.9$ \\
\hline Repair time distribution & Range of $\mu$ & Rank \\
\hline Exponential $(M)$ & $0.06<\mu<0.16$ & Model 2 \\
3-stage Erlang $\left(E_{3}\right)$ & $0.06<\mu<0.16$ & Model 2 \\
Deterministic $(D)$ & $0.06<\mu<0.16$ & Model 2 \\
Lognormal $(L N)$ & $0.06<\mu<0.16$ & Model 2 \\
Weibull $(W)$ & $0.06<\mu<0.16$ & Model 2 \\
\hline
\end{tabular}


Table 7. Rank of $C_{i} / A v_{i}$ for $\lambda=0.001, \alpha=0.0005, \mu=0.1, c=0.9$

\begin{tabular}{lll}
\hline Repair time distribution & Range of $\beta$ & Rank \\
\hline Exponential $(M)$ & $1<\beta<10$ & Model 2 \\
3-stage Erlang $\left(E_{3}\right)$ & $1<\beta<10$ & Model 2 \\
Deterministic $(D)$ & $1<\beta<10$ & Model 2 \\
Lognormal $(L N)$ & $1<\beta<10$ & Model 2 \\
Weibull $(W)$ & $1<\beta<10$ & Model 2 \\
\hline
\end{tabular}

Table 8. Rank of $C_{i} / A v_{i}$ for $\lambda=0.001, \alpha=0.0005, \mu=0.1, \beta=2.4$

\begin{tabular}{lll}
\hline Repair time distribution & Range of $c$ & Rank \\
\hline Exponential $(M)$ & $0.5<c<1.0$ & Model 2 \\
3-stage Erlang $\left(E_{3}\right)$ & $0.5<c<1.0$ & Model 2 \\
Deterministic $(D)$ & $0.5<c<1.0$ & Model 2 \\
Lognormal $(L N)$ & $0.5<c<1.0$ & Model 2 \\
Weibull $(W)$ & $0.5<c<1.0$ & Model 2 \\
\hline
\end{tabular}

Table 9. The CPU mean time of 100 computation times spent for calculating availabilities when $\lambda$ changes from 0.001 to 0.01 .

\begin{tabular}{lllr}
\hline Repair time distribution & Original Method & Reciprocal transformation & Ratio $(\%)$ \\
\hline Exponential $(M)$ & 0.01579 second & 0.12328 second & $-680.7 \%$ \\
3-stage Erlang $\left(E_{3}\right)$ & 0.02077 second & 0.37531 second & $-1706 \%$ \\
Deterministic $(D)$ & 0.00531 second & 0.00501 second & $5.65 \%$ \\
Lognormal $(L N)$ & 8.12843 second & 2.81016 second & $65.43 \%$ \\
Weibull $(W)$ & 2.14001 second & 2.60921 second & $-21.93 \%$ \\
\hline
\end{tabular}

Table 10. The CPU mean time of 100 computation times spent for calculating availabilities when $\mu$ changes from 0.06 to 0.16 .

\begin{tabular}{lrlr}
\hline Repair time distribution & Original Method & Reciprocal transformation & Ratio $(\%)$ \\
\hline Exponential $(M)$ & 0.09325 second & 0.11281 second & $-20.98 \%$ \\
3-stage Erlang $\left(E_{3}\right)$ & 0.01999 second & 0.35078 second & $-1655 \%$ \\
Deterministic $(D)$ & 0.005 second & 0.00515 second & $-3 \%$ \\
Lognormal $(L N)$ & 8.69878 second & 2.36954 second & $72.76 \%$ \\
Weibull $(W)$ & 2.5014 second & 2.90935 second & $-16.31 \%$ \\
\hline
\end{tabular}

Table 11. The CPU mean time of 100 computation times spent for calculating availabilities when $\beta$ changes from 1.0 to 10 .

\begin{tabular}{llcr}
\hline Repair time distribution & Original Method & Reciprocal transformation & Ratio $(\%)$ \\
\hline Exponential $(M)$ & 0.00687 second & 0.0875 second & $-1174 \%$ \\
3-stage Erlang $\left(E_{3}\right)$ & 0.01187 second & 0.12266 second & $-933 \%$ \\
Deterministic $(D)$ & 0.01015 second & 0.00985 second & $2.96 \%$ \\
Lognormal $(L N)$ & 2.66095 second & 0.0936 second & $96.48 \%$ \\
Weibull $(W)$ & 0.01641 second & 0.01655 second & $-0.09 \%$ \\
\hline
\end{tabular}


Table 12. The CPU mean time of 100 computation times spent for calculating availabilities when $c$ changes from 0.5 to 1.0 .

\begin{tabular}{lrlr}
\hline Repair time distribution & Original Method & Reciprocal transformation & Ratio $(\%)$ \\
\hline Exponential $(M)$ & 0.01187 second & 0.04937 second & $-315.9 \%$ \\
3-stage Erlang $\left(E_{3}\right)$ & 0.01703 second & 0.13703 second & $-704.6 \%$ \\
Deterministic $(D)$ & 0.01173 second & 0.00733 second & $38.51 \%$ \\
Lognormal $(L N)$ & 4.04488 second & 0.28375 second & $93.98 \%$ \\
Weibull $(W)$ & 0.0397 second & 0.02594 second & $34.66 \%$ \\
\hline
\end{tabular}

\section{THE EFFICIENCY OF RECIPROCAL TRANSFORMATION METHOD}

To investigate the efficiency of computation of the reciprocal transformation method, the CPU computation time spent for calculating availabilities of three models for five different repair time distributions is recorded. Numerical results of the mean CPU time of 100 computation times using original method and the reciprocal transformation method are shown in Tables 9-12. Noting that the computation of original method indicates the MAPLE integral code used with lower bound zero and upper bound infinity. The percentages in the last column denote the relative improvement (worsening) of the computation time using reciprocal transformation.

From Tables 9-12, it can be observed that the reciprocal transformation significantly reduces the CPU computation time when the repair time distribution is Lognormal. Alternatively, the reciprocal transformation to the $M$ and $E_{3}$ distributions doesn't bring out the decreasing of computation time due to the explicitly closed-form with simple computation.

\section{REFERENCES}

1. K. S. Trivedi, Probability and Statistics with Reliability, Queueing and Computer Science Applications, Second Edition, John Wiley and Sons, Inc. New York, 2002.

2. G. Casella and R. L. Berger, Statistical Inference, 2nd ed., Duxbury Inc, 2002.

3. K.-H. Wang and L. W. Chiu, Cost benefit analysis of availability systems with warm standby units and imperfect coverage, Applied Mathematics and Computation 172 (2) 1239-1256, 2006.

4.K.-H. Wang and Y.-J. Chen Y.-J, Comparative Analysis of Availability between Three System with General Repair Times, Reboot Delay and Switching Failures, Applied Mathematics and Computation 215(1) 384-394, 2009

5. J.-C. Ke, Z.-L. Su and K.-H. Wang, An Availability System with General Repair Distribution: Statistical Inference, Communications in Statistics-Theory and Methods 38(9) 1508-1525, 2009. 